Tohoku Math. J

58 (2006), 149-159

\title{
SOME REMARKS ON WEAK COMPACTNESS IN THE DUAL SPACE OF A JB*-TRIPLE
}

\author{
Antonio M. Peralta* \\ (Received March 30, 2004, revised January 18, 2005)
}

\begin{abstract}
We obtain several characterizations of relatively weakly compact subsets in the predual of a $\mathrm{JBW}^{*}$-triple. As a consequence, we describe the relatively weakly compact subsets in the predual of a JBW*-algebra.
\end{abstract}

Introduction. The study of relatively weakly compact subsets of the predual of a von Neumann algebra is mainly due to Takesaki [24], Akemann [2], Akemann, Dodds and Gamlen [3] and Saitô [22]. Their results on characterizations of relatively weakly compact subsets in the predual of a von Neumann algebra were the key tool for the description of weakly compact operators from a $C^{*}$-algebra to a complex Banach space found by Jarchow $[17,18]$

Every von Neumann algebra belongs to a more general class of Banach spaces known as $\mathrm{JBW}^{*}$-triples. A JB*-triple is a complex Banach space equipped with a Jordan triple product satisfying some algebraic and geometric properties (see the definition below). JB*-triples were introduced by Kaup [19] in the study of bounded symmetric domains in complex Banach spaces. The class of $\mathrm{JB}^{*}$-triples contains all $\mathrm{C}^{*}$-algebras and all $\mathrm{JB}^{*}$-algebras. A JBW*-triple is a $\mathrm{JB}^{*}$-triple which is also a dual Banach space; thus every von Neumann algebra is a $\mathrm{JBW}^{*}$ triple.

The study of weakly compact operators from a JB*-triple to a Banach space was developed in [9] and [21, Theorem 10 and the succedent remarks]. However, contrary to the case of $\mathrm{C}^{*}$-algebras, the characterization of weakly compact operators from a $\mathrm{JB}^{*}$-triple to a complex Banach space was not obtained by describing the relatively weakly compact subsets of the predual of a JBW*-triple. The objective of this paper is to describe the relatively weakly compact subsets in the predual of a JBW*-triple. Theorem 1.1 and Corollary 1.4 generalize the classical description of relatively weakly compact subsets in the predual of a von Neumann algebra to the setting of $\mathrm{JBW}^{*}$-triple preduals. The above results are specialized to $\mathrm{JBW}^{*}$-algebra preduals in Theorem 1.5.

As a consequence of our results, we prove that for every norm bounded sequence $\left(\phi_{n}\right)$ in the predual of a $\mathrm{JBW}^{*}$-triple $W$, for each norm-one functional $\varphi \in W_{*}$ and for every $\varepsilon>0$, there exists a tripotent $e \in W$ such that $\varphi(e)>1-\varepsilon$ and $\left(\phi_{n}\right)$ admits a subsequence

2000 Mathematics Subject Classification. Primary 17C65; Secondary 46L70, 46L05, 47B99.

Key words and phrases. JB*-triples, JB*-álgebras, weak compactness.

* Partially supported by D.G.I. project no. MTM 2005-02541, and Junta de Andalucía grant FQM 0199. 
which converges weakly to a functional in $\left(W_{2}(e)_{*}\right.$, where $W_{2}(e)$ is the Peirce 2-subspace associated to $e$. This result extends [7] to the setting of $\mathrm{JBW}^{*}$-triples.

Let $X$ be a Banach space. Throughout the paper, $B_{X}$ and $X^{*}$ denote the closed unit ball of $X$ and the dual space of $X$, respectively. If $X$ is a dual Banach space, $X_{*}$ will stand for a predual of $X$.

1. Weakly compact sets in the dual of a $\mathbf{J B}^{*}$-triple. A $J B^{*}$-triple is a complex Banach space $E$ equipped with a continuous triple product

$$
\begin{gathered}
\{., ., .\}: E \times E \times E \rightarrow E \\
(x, y, z) \mapsto\{x, y, z\},
\end{gathered}
$$

which is bilinear and symmetric in the outer variables and conjugate linear in the middle one and satisfies:

(a) (Jordan Identity)

$$
L(x, y)\{a, b, c\}=\{L(x, y) a, b, c\}-\{a, L(y, x) b, c\}+\{a, b, L(x, y) c\}
$$

for all $x, y, a, b, c \in E$, where $L(x, y): E \rightarrow E$ is the linear mapping given by $L(x, y) z=$ $\{x, y, z\}$

(b) The map $L(x, x)$ is an hermitian operator with non-negative spectrum for all $x \in E$;

(c) $\|\{x, x, x\}\|=\|x\|^{3}$ for all $x \in E$.

Every $\mathrm{C}^{*}$-algebra is a $\mathrm{JB}^{*}$-triple with respect to the triple product

$$
\{x, y, z\}=2^{-1}\left(x y^{*} z+z y^{*} x\right) .
$$

Every $\mathrm{JB}^{*}$-algebra is a $\mathrm{JB}^{*}$-triple with triple product given by

$$
\{a, b, c\}=\left(a \circ b^{*}\right) \circ c+\left(c \circ b^{*}\right) \circ a-(a \circ c) \circ b^{*} .
$$

The Banach space $B(H, K)$ of all bounded linear operators between two complex Hilbert spaces $H, K$ is also an example of a $\mathrm{JB}^{*}$-triple with product $\{R, S, T\}=2^{-1}\left(R S^{*} T+T S^{*} R\right)$.

A $\mathrm{JBW}^{*}$-triple is a $\mathrm{JB}^{*}$-triple which is also a dual Banach space. The bidual, $E^{* *}$, of every $\mathrm{JB}^{*}$-triple, $E$, is a $\mathrm{JBW}^{*}$-triple with triple product extending the product of $E$ (cf. [11]).

Let $E$ be a $\mathrm{JB}^{*}$-triple. An element $e \in E$ is said to be a tripotent if $\{e, e, e\}=e$. The set of all tripotents of $E$ is denoted by $\operatorname{Tri}(E)$. Given a tripotent $e \in E$, there exists a decomposition of $E$ in terms of the eigenspaces of $L(e, e)$ given by

$$
E=E_{0}(e) \oplus E_{1}(e) \oplus E_{2}(e),
$$

where $E_{k}(e):=\{x \in \mathcal{E} ; L(e, e) x=(k / 2) x\}$ is a subtriple of $E(k: 0,1,2)$. The natural projection of $E$ onto $E_{k}(e)$ will be denoted by $P_{k}(e)$. The following rules are also satisfied:

$$
\begin{gathered}
\left\{E_{k}(e), E_{l}(e), E_{m}(e)\right\} \subseteq E_{k-l+m}(e), \\
\left\{E_{0}(e), E_{2}(e), E\right\}=\left\{E_{2}(e), E_{0}(e), E\right\}=0,
\end{gathered}
$$

where $E_{k-l+m}(e)=0$ whenever $k-l+m$ is not in $\{0,1,2\}$. It is also known that $E_{2}(e)$ is a unital $\mathrm{JB}^{*}$-algebra with respect to the product and involution given by $x \circ y=\{x, e, y\}$ and $x^{*}=\{e, x, e\}$, respectively. When $E$ is a JBW*-triple, $E_{2}(e)$ is a JBW*-algebra. 
For background materials about JB- and JBW-algebras the reader is referred to [14]. We recall that JB-algebras (resp. JBW-algebras) are nothing but the self-adjoint parts of JB*algebras (resp. JBW*-algebras) [26] (resp. [12]).

Two tripotents $e, f$ in a $\mathrm{JB}^{*}$-triple $\operatorname{Tri}(E)$ are said to be orthogonal if $e$ belongs to $E_{0}(f)$ and $f$ belongs to $E_{0}(e)$. Let $e, f \in E$. Following [20, §5], we say that $e \leq f$ if and only if $f-e$ is a tripotent which is orthogonal to $e$. It is also known that $e \leq f$ if and only if $e$ is a symmetric projection in $E_{2}(f)$.

Let $W$ be a $\mathrm{JBW}^{*}$-triple and $\varphi$ a norm-one element in $W_{*}$. Let $z$ be a norm-one element in $W$ such that $\varphi(z)=1$. By [4] the mapping $(x, y) \mapsto \varphi\{x, y, z\}$ defines a positive sesquilinear form on $W$ which does not depend on the element $z$. Thus the law $x \mapsto\|x\|_{\varphi}:=$ $(\varphi\{x, x, z\})^{1 / 2}, x \in W$, defines a prehilbert seminorm on $W$. If $E$ is a $\mathrm{JB}^{*}$-triple and $\varphi$ is a norm-one element in $E^{*}$, then $\|\cdot\|_{\varphi}$ is a prehilbertian seminorm on $E^{* *}$ and hence on $E$. The strong*-topology of $W$, introduced by Barton and Friedman in [5], is the topology on $W$ generated by the family of seminorms $\left\{\|\cdot\|_{\varphi} ; \varphi \in S_{W_{*}}\right\}$. We use the symbol $S^{*}\left(W, W_{*}\right)$ to denote the strong*-topology of $W$. When $\varphi_{1}, \varphi_{2}$ are two norm-one functionals in $W_{*}$, then we write $\|\cdot\|_{\varphi_{1}, \varphi_{2}}$ for the hilbertian semi-norm defined by

$$
\|x\|_{\varphi_{1}, \varphi_{2}}^{2}:=\|x\|_{\varphi_{1}}^{2}+\|x\|_{\varphi_{2}}^{2} .
$$

If $A$ is a $\mathrm{JBW}^{*}$-algebra regarded as a $\mathrm{JB}^{*}$-triple, then the $S^{*}\left(A, A_{*}\right)$ coincides with the algebra strong*-topology of $A$ generated by all the seminorms of the form $x \mapsto \sqrt{\phi\left(x \circ x^{*}\right)}$, where $\phi$ is any normal state in $A$. Consequently, when a von Neumann algebra $M$ is regarded as a $\mathrm{JBW}^{*}$-triple, the $S^{*}\left(M, M_{*}\right)$ coincides with the strong*-topology on $M$ (see [23, Definition 1.8.7]).

A JB*-triple $E$ is said to be abelian if for every $x, y, a, b \in E$, the operators $L(x, y)$ and $L(a, b)$ commute. Every abelian $\mathrm{JBW}^{*}$-triple is a triple isomorphic (and hence isometric) to a von Neumann algebra.

Let $W$ be a $\mathrm{JBW}^{*}$-triple with predual $W_{*}$. Since the triple product of $W$ is separately weak*-continuous (cf. [6]), every maximal abelian subtriple is weak*-closed and hence a $\mathrm{JBW}^{*}$-subtriple of $W$.

THEOREM 1.1. Let $W$ be a JBW*-triple with predual $W_{*}$ and let $K$ be a subset in $W_{*}$. Then the following are equivalent:

(a) $K$ is relatively weakly compact.

(b) There exist norm-one elements $\varphi_{1}, \varphi_{2} \in W_{*}$ with the following property: Given $\varepsilon>0$, there exists $\delta>0$ such that for every $x \in W$ with $\|x\| \leq 1$ and $\|x\|_{\varphi_{1}, \varphi_{2}}<\delta$, we have $|\phi(x)|<\varepsilon$ for each $\phi \in K$.

(c) The restriction $\left.K\right|_{C}$ of $K$ to each maximal abelian subtriple $C$ of $W$ is relatively $\sigma\left(C_{*}, C\right)$-compact.

Proof. (a) $\Rightarrow$ (b) We assume that $K \subset W_{*}$ is relatively weakly compact. We may also assume that $K \subseteq B_{W_{*}}$. Let us fix $\varepsilon>0$. Let $D=\overline{\mid c o}^{w}(K)$ denote the weakly closed absolutely convex hull of $K$ in $W_{*}$. Then $D$ is an absolutely convex weakly compact subset 
of $W_{*}$. Let $Y$ denote the Banach space $\ell_{1}(D)$ and $F$ the bounded linear operator from $Y$ to $W_{*}$ given by

$$
F\left(\left\{\lambda_{\varphi}\right\}_{\varphi \in D}\right):=\sum_{\varphi \in D} \lambda_{\varphi} \varphi .
$$

Clearly, $F\left(B_{Y}\right)=D$. Since $D$ is weakly compact, $F$ (and hence $F^{*}$ ) is a weakly compact operator. By [21, Theorem 10] there exist norm-one elements $\varphi_{1}, \varphi_{2} \in W_{*}$ and a function $N:(0,+\infty) \rightarrow(0,+\infty)$ such that

$$
\left\|F^{*}(x)\right\| \leq N(\varepsilon)\|x\|_{\varphi_{1}, \varphi_{2}}+\varepsilon\|x\|
$$

for all $x \in W$ and $\varepsilon>0$.

Let $x \in W$. It is clear that

$$
\begin{aligned}
\sup _{\phi \in D}|\phi(x)| & =\sup _{y \in B_{Y}}|F(y)(x)|=\sup _{y \in B_{Y}}\left|F^{*}(x)(y)\right| \leq\left\|F^{*}(x)\right\| \\
& \leq N\left(\frac{\varepsilon}{2}\right)\|x\|_{\varphi_{1}, \varphi_{2}}+\frac{\varepsilon}{2}\|x\| .
\end{aligned}
$$

Finally, taking $\delta=N(\varepsilon / 2)^{-1} \cdot \varepsilon / 2$, we conclude that for every $x \in W$ with $\|x\| \leq 1$ and $\|x\|_{\varphi_{1}, \varphi_{2}} \leq \delta$ we have $|\phi(x)| \leq \varepsilon$ for each $\phi \in K$.

(b) $\Rightarrow$ (c) Suppose that there exists a maximal abelian subtriple $C$ of $W$ such that $\left.K\right|_{C}$ is not relatively $\sigma\left(C_{*}, C\right)$-compact. Since $C$ is a maximal abelian subtriple, $C$ is weak*-closed and thus is isomorphic (and hence isometric) to a von Neumann algebra, provided the latter is considered as a JB*-triple. By [2, Theorem II.2] (see also [25, Theorem 5.4]) there exists an orthogonal sequence $\left(p_{n}\right)$ of symmetric projections in $C$ and a sequence $\left(\varphi_{n}\right) \subseteq K$ satisfying

$$
\left|\varphi_{n}\left(p_{n}\right)\right| \geq \Theta>0
$$

By hypothesis, there are norm-one elements $\varphi_{1}, \varphi_{2}$ in $W_{*}$ and $\delta>0$ such that for every $x \in W$ with $\|x\| \leq 1$ and $\|x\|_{\varphi_{1}, \varphi_{2}}<\delta$, we have $|\phi(x)|<\Theta / 2$ for each $\phi \in K$.

Let $\psi$ be a normal state of $C$. Since $\psi\left(p_{n} p_{n}^{*}+p_{n}^{*} p_{n}\right)=2 \psi\left(p_{n}\right)$ tends to zero, it follows that $\left(p_{n}\right)$ is a strong*-null sequence in $C$. By [8, Corollary] we conclude that $\left(p_{n}\right) \rightarrow 0$ in the $\mathrm{S}^{*}\left(W, W_{*}\right)$-topology of $W$. In particular, $\left\|p_{n}\right\|_{\varphi_{1}, \varphi_{2}} \rightarrow 0$. Therefore, there exists $N \in N$ such that for every $n \in N, n \geq N$, we have

$$
\left\|p_{n}\right\|_{\varphi_{1}, \varphi_{2}}<\delta
$$

As a consequence, $\left|\phi\left(p_{n}\right)\right|<\Theta / 2$ for each $\phi \in K$, which contradicts (2).

(c) $\Rightarrow$ (a) Suppose that the restriction $\left.K\right|_{C}$ of $K$ to each maximal abelian subtriple $C$ of $W$ is relatively $\sigma\left(C_{*}, C\right)$-compact. Let $x \in W$. Since the $\mathrm{JBW}^{*}$-subtriple of $W$ generated by $x$ is abelian, by Zorn's Lemma there exists a maximal abelian subtriple $C$ of $W$ containing $x$. By hypothesis, $\left.K\right|_{C}$ is relatively $\sigma\left(C_{*}, C\right)$-compact, and hence $\{\phi(x) ; \phi \in K\}$ is bounded. It follows from the uniform boundedness theorem that $K$ is bounded. Let $\widetilde{K}$ denote the $\sigma\left(W^{*}, W\right)$-closure of $K$ in $W^{*}$. Since $K$ is bounded, $\widetilde{K}$ is $\sigma\left(W^{*}, W\right)$-compact. 
We claim $\widetilde{K} \subset W_{*}$. Indeed, let $\phi \in \widetilde{K}$. Let $C$ be any maximal abelian subtriple of $W$. Then $\left.\phi\right|_{C}$ is in the $\sigma\left(C^{*}, C\right)$-closure of $\left.K\right|_{C}$. By assumptions, $\left.K\right|_{C}$ is relatively $\sigma\left(C_{*}, C\right)$ compact and thus $\left.\phi\right|_{C} \in C_{*}$. Now, by [15, Theorem 3.23], it follows that $\phi \in W_{*}$ as claimed.

Since $\widetilde{K} \subset W_{*}, \widetilde{K}$ coincides with the $\sigma\left(W_{*}, W\right)$-closure of $K$ in $W$, and hence $K$ is relatively $\sigma\left(W_{*}, W\right)$-compact.

The following corollary extends [10, Lemma 4] (see also [1, Lemma 1]) to general $\mathrm{JBW}^{*}$-triples, and it is in fact a natural extension of [25, Lemma III.5.5] to the setting of $\mathrm{JBW}^{*}$-triples.

COROLlaRY 1.2. Let $W$ be a JBW*-triple. Let $\left(\varphi_{k}\right)$ be a weakly convergent sequence in $W_{*}$ and $\left(x_{n}\right)$ a strong-*-null sequence in $W$. Then

$$
\lim _{n \rightarrow+\infty} \sup _{k \in N}\left|\varphi_{k}\left(x_{n}\right)\right|=0 .
$$

Proof. Suppose that $\left(\varphi_{k}\right) \rightarrow \varphi$ weakly in $W_{*}$. The set $K=\left\{\varphi_{k} ; k \in N\right\}$ is a relative weakly compact subset of $W_{*}$ by the Eberlein-Smulian theorem. Let $\varepsilon>0$. By Theorem 1.1, there are norm-one elements $\varphi_{1}, \varphi_{2} \in W_{*}$ and $\delta>0$ such that for every $x \in W$ with $\|x\| \leq 1$ and $\|x\|_{\varphi_{1}, \varphi_{2}}<\delta$, we have $|\phi(x)|<\varepsilon$ for each $\phi \in K$. Since $\left(x_{n}\right)$ is strong*-null, there exists $N \in N$ such that for every $n \geq N$ it follows that $\left\|x_{n}\right\| \varphi_{1}, \varphi_{2} \leq \delta$. Thus, for every $n \geq N$, we have $\left|\varphi\left(x_{n}\right)\right| \leq \varepsilon$ for all $f \in K$.

REMARK 1.3. Let $W$ be a JBW*-triple. Suppose that $K \in W_{*}$ is a relatively weakly compact set. Then, similar arguments to those given in the proof of Corollary 1.2 show that for each strong*-null sequence $\left(x_{n}\right)$ in $W$ we have

$$
\lim _{n \rightarrow+\infty} \varphi\left(x_{n}\right)=0
$$

uniformly for $\varphi \in K$.

Using Theorem 1.1, we now generalize to the setting of JBW*-triples some known characterizations of weak compactness in the predual of a $\mathrm{W}^{*}$-algebra (compare [25, Theorem 5.4]).

COROLlary 1.4. Let $K$ be a bounded subset in the predual of a JBW*-triple $W$. Then the following assertions are equivalent:

(a) $K$ is relatively weakly compact.

(b) The restriction of $K$ to $W_{2}(e)$ is relatively $\sigma\left(\left(W_{2}(e)\right)_{*}, W_{2}(e)\right)$-compact in $\left(W_{2}(e)\right)_{*}$ for every tripotent $e \in W$.

(c) For any monotone decreasing sequence of tripotents $\left(e_{n}\right)$ in $W$ with $\left(e_{n}\right) \rightarrow 0$ in the weak*-topology, we have $\lim _{n \rightarrow+\infty} \phi\left(e_{n}\right)=0$ uniformly for $\phi \in K$.

Proof. (a) $\Rightarrow$ (b) Suppose $K$ is relatively weakly compact in $W_{*}$. Let $e$ be a tripotent in $W$. Since the map: $\left.\phi \mapsto \phi\right|_{W_{2}(e)}$ is a weakly continuous operator from $W_{*}$ to $\left(W_{2}(e)\right)_{*}$, it follows that $\left.K\right|_{W_{2}(e)}$ is relatively $\sigma\left(\left(W_{2}(e)\right)_{*}, W_{2}(e)\right)$-compact in $\left(W_{2}(e)\right)_{*}$. 
(b) $\Rightarrow$ (c) Let $\left(e_{n}\right)$ be a monotone decreasing sequence in $W$ with $\left(e_{n}\right) \rightarrow 0$ in the $\sigma\left(W, W_{*}\right)$-topology. Since for each $n \in N$, we have $e_{1} \geq e_{n}$, it follows that $\left(e_{n}\right)$ is a monotone decreasing sequence of projections in $W_{2}\left(e_{1}\right)$ with $\left(e_{n}\right) \rightarrow 0$ in the $\sigma\left(W_{2}\left(e_{1}\right),\left(W_{2}\left(e_{1}\right)\right)_{*}\right)$-topology. It is not hard to see that $\left(e_{n}\right) \rightarrow 0$ in the strong-* topology of $W_{2}\left(e_{1}\right)$. Since, by assumptions, $\left.K\right|_{W_{2}\left(e_{1}\right)}$ is relatively $\sigma\left(\left(W_{2}\left(e_{1}\right)\right)_{*}, W_{2}\left(e_{1}\right)\right)$-compact, we conclude from Remark 1.3 that $\lim _{n \rightarrow+\infty} \phi\left(e_{n}\right)=0$ uniformly for $\phi \in K$.

(c) $\Rightarrow$ (a) To obtain a contradiction, suppose that $K$ is not relatively weakly compact. By Theorem 1.1 there exists a maximal abelian $\mathrm{JBW}^{*}$-subtriple $C$ of $W$ such that $\left.K\right|_{C}$ is not relatively $\sigma\left(C_{*}, C\right)$-compact. As remarked above, $C$ is a triple isomorphic to an abelian von Neumann algebra, provided the latter is regarded as a JBW*-triple. By [25, Theorem 5.4] there exists a monotone decreasing sequence $\left(p_{n}\right)$ of projections in $C$ with $\left(p_{n}\right) \rightarrow 0$ in the $\sigma\left(C, C_{*}\right)$-topology and $\lim _{n \rightarrow+\infty} \phi\left(p_{n}\right) \neq 0$ uniformly for $\left.\phi \in K\right|_{C}$. Therefore there exists a monotone decreasing sequence $\left(p_{n}\right)$ of tripotents in $W$ with $\left(p_{n}\right) \rightarrow 0$ in the weak*-topology of $W$ and $\lim _{n \rightarrow+\infty} \phi\left(p_{n}\right) \neq 0$ uniformly for $\phi \in K$, which is a contradiction.

We do not know if the semi-norm $\|\cdot\|_{\varphi_{1}, \varphi_{2}}$ appearing in Theorem 1.1 (b) could be replace by a semi-norm of the form $\|\cdot\|_{\varphi}$ for a suitable norm-one functional $\varphi \in W_{*}$. This problem is connected with a problem on Grothendieck's inequalities for JB*-triples (compare [21, Remark 3]). We next show a positive answer to the above problem in the particular case of a $\mathrm{JBW}^{*}$-algebra.

Let $M$ be a JBW*-algebra with predual $M_{*}$. Let $\varphi_{1}, \varphi_{2}$ be two norm-one functionals in $M_{*}$. For each $i \in\{1,2\}$ we take a tripotent $e_{i} \in M$ such that $\varphi_{i}\left(e_{i}\right)=1$. Let $\psi_{i}$ denote the norm-one functional in $M_{*}$ given by $\psi_{i}(x):=\varphi_{i}\left(x \circ e_{i}\right)$ for any $x \in M$. From the expression

$$
\left\{x, x, e_{i}\right\}+\left\{x^{*}, x^{*}, e_{i}\right\}=2 e_{i} \circ\left(x \circ x^{*}\right),
$$

we conclude that $\psi_{i}$ is a positive normal state of $M$. Moreover, the identity

$$
\|x\|_{\varphi_{i}}^{2}+\left\|x^{*}\right\|_{\varphi_{i}}^{2}=2 \psi_{i}\left(x \circ x^{*}\right)=2\|x\|_{\psi_{i}}^{2}
$$

holds for all $x \in M$. Set $\psi=1 / 2\left(\psi_{1}+\psi_{2}\right)$. Then $\psi$ is a normal state of $M$ satisfying

$$
\|x\|_{\varphi_{1}, \varphi_{2}} \leq 2\|x\|_{\psi}
$$

for all $x \in M$. We can now reformulate Theorem 1.1 to the setting of $\mathrm{JBW}^{*}$-algebras.

THEOREM 1.5. Let $M$ be a $J B W^{*}$-algebra. Let $K$ be a norm bounded subset in $M_{*}$. Then the following assertions are equivalent:

(a) $K$ is relatively weakly compact.

(b) The restriction $\left.K\right|_{C}$ of $K$ to each maximal associative subalgebra $C$ of $M$ is relatively $\sigma\left(C_{*}, C\right)$-compact.

(c) There exists a normal state $\psi \in M_{*}$ with the following property: Given $\varepsilon>0$, there exists $\delta>0$ such that for every $x \in W$ with $\|x\| \leq 1$ and $\|x\|_{\psi}<\delta$, we have $|\phi(x)|<\varepsilon$ for each $\phi \in K$.

(d) For any monotone decreasing sequence of projections $\left(e_{n}\right)$ in W with $\left(e_{n}\right) \rightarrow 0$ in the weak $*$-topology, we have $\lim _{n \rightarrow+\infty} \phi\left(e_{n}\right)=0$ uniformly for $\phi \in K$. 
2. Applications. Let $\left(\phi_{n}\right)$ be a bounded sequence in the predual of a JBW*-triple $W$. It is known that, in general, $\left(\phi_{n}\right)$ needs not admit a weakly convergent subsequence. In the setting of von Neumann algebras we can say more about bounded sequences of normal functionals. Indeed, in a recent paper, Brooks, Saitô and Wright [7] have shown that each bounded sequence in the predual of a von Neumann algebra has a subsequence which is nearly weakly convergent. More concretely, for each bounded sequence $\left(\phi_{n}\right)$ in the predual of a von Neumann algebra $M$, for each normal state $\psi$ and for each $\varepsilon>0$, there exists a projection $e \in M$ such that $\psi(1-e) \leq \varepsilon$ and the restriction of $\left(\phi_{n}\right)$ to $e M e$ has a subsequence which converges weakly to a normal functional on $e M e$. The aim of this section is to obtain an analogue of the above fact in the setting of JBW*-triples.

The following lemma provides sufficient conditions to assure relative weak compactness in the predual of a $\mathrm{JBW}^{*}$-triple. It is also a natural extension of [7, Lemma 2] to the setting of $\mathrm{JBW}^{*}$-triples.

LEMmA 2.1. Let $\left(\phi_{n}\right)$ be a bounded sequence in the predual of a JBW*-triple W. Let $\varphi$ be a norm-one element in $W_{*}$ such that the following property holds; for each $c>0$ there exists $\eta>0$ such that for every tripotent $e \in W$ with $\|e\|_{\varphi}<\eta$, the set

$$
\left\{m \in N \text {; there exists } u \in \operatorname{Tri}(W) \text { with } u \leq e \text { and }\left|\phi_{m}(u)\right| \geq c\right\}
$$

is finite. Then $\left\{\phi_{n} ; n \in N\right\}$ is relatively weakly compact in $W_{*}$.

PROOF. Let $\left(e_{n}\right)$ be a weak*-null, monotone decreasing sequence of tripotents in $W$. Let $c>0$ and let $\eta>0$ be the positive number given by the property stated above.

Since for each $n \in N, e_{1} \geq e_{n}$, we conclude that $\left(e_{n}\right)$ is a weak*-null, monotone decreasing sequence of projections in $W_{2}\left(e_{1}\right)$. As remarked in the above section, it is not hard to see that $\left(e_{n}\right)$ is strong*-null in $W_{2}(e)$ and from [8, Corollary], $\left(e_{n}\right)$ is strong*-null in $W$. In particular, $\left\|e_{n}\right\|_{\varphi} \rightarrow 0$. Then there exists $m_{1} \in N$ such that for each $n \geq m_{1}$ we have $\left\|e_{n}\right\|_{\varphi}<\eta$. Since the set

$$
\left\{m \in N ;\left|\phi_{m}\left(e_{n}\right)\right| \geq c \text { for some } n \geq m_{1}\right\}
$$

is finite by hypothesis, we conclude that there exists $m_{0} \in N$ such that for each $m \geq m_{0}$ we have $\left|\phi_{m}\left(e_{n}\right)\right|<c$ for each $n \geq m_{1}$.

Since for each $1 \leq j \leq m_{0}$ the sequence $\left(\phi_{j}\left(e_{n}\right)\right)_{n \in N}$ tends to zero, we deduce that there exists $m_{2} \in N$ such that for each $n \geq m_{2}$ and $1 \leq j \leq m_{0}$ we have $\left|\phi_{j}\left(e_{n}\right)\right|<c$. Therefore, for each $n \geq \max \left\{m_{1}, m_{2}\right\}$, we have $\left|\phi_{m}\left(e_{n}\right)\right|<c$ for all $m \in N$. Corollary 1.4 then yields the desired statement.

When, in the proof of Lemma 2.1, Theorem 1.5 replaces Corollary 1.4, we obtain the following

LEMMA 2.2. Let $M$ be a JBW*-algebra and $\left(\phi_{n}\right)$ a bounded sequence in $M_{*}$. Let $\varphi$ be a normal state of $M$ such that the following property holds; for each $c>0$ there exists $\eta>0$ such that for every projection $e \in M$ with $\|e\|_{\varphi}<\eta$, the set

$\left\{m \in N\right.$; there exists a projection $p \in M$ with $p \leq e$ and $\left.\left|\phi_{m}(p)\right| \geq c\right\}$ 
is finite. Then $\left\{\phi_{n} ; n \in N\right\}$ is relatively weakly compact in $M_{*}$.

Let $M$ be a JBW*-algebra. Let $\varphi$ be a positive normal functional on $M$ and $\left(\phi_{n}\right)$ a normbounded sequence in $M_{*}$. We shall denote by $\Delta$ the set of all $c \in \boldsymbol{R}^{+}$such that for each $\eta>0$ there exists a projection $e_{\eta} \in W$ such that $\left\|e_{\eta}\right\|_{\varphi}<\eta$ and the set

$$
\left\{m \in N \text {; there exists a projection } p \in M \text { with } p \leq e_{\eta} \text { and }\left|\phi_{m}(p)\right| \geq c\right\}
$$

is infinite. Following [7, Definition in page 162], we call $\Delta$ the anti-compactness set of $\left(\phi_{n}\right)$ with respect to the functional $\varphi$. It is clear that $\Delta$ is bounded.

REMARK 2.3. Let $M$ be a JBW*-algebra. Let $\varphi$ be a positive functional in $M_{*},\left(\phi_{n}\right)$ a norm-bounded sequence in $M_{*}$, and $\Delta$ the anti-compactness set of $\left(\phi_{n}\right)$ with respect to $\varphi$. We claim that $\left(\phi_{n}\right)$ is relatively weakly compact in $M_{*}$ whenever $\Delta=\emptyset$. Indeed, let $c \in \boldsymbol{R}^{+}$. Since $c \notin \Delta$, there exists $\eta>0$ such that for every projection $e \in M$ with $\|e\|_{\varphi}<\eta$, the set

$$
\left\{m \in N \text {; there exists a projection } p \in M \text { with } p \leq e \text { and }\left|\phi_{m}(p)\right| \geq c\right\}
$$

is finite. We conclude from Lemma 2.2 that $\left(\phi_{n}\right)$ is relatively weakly compact in $W_{*}$.

We recall that a positive functional $\psi$ of a $\mathrm{JB}^{*}$-algebra $A$ is said to be faithful if and only if $\psi(x)>0$ for every positive element $x \in A \backslash\{0\}$. Suppose that a JBW*-algebra $M$ has a faithful normal state $\psi$. Then the strong*-topology in the closed unit ball of $M$ is metrized by the distance

$$
d_{\psi}(a, b):=\left(\psi\left((a-b) \circ(a-b)^{*}\right)\right)^{1 / 2} .
$$

More precisely, a bounded net $\left(x_{i}\right)_{i \in I}$ in $M$ converges in the strong*-topology of $M$ to an element $x \in M$ if and only if $d_{\psi}\left(x_{i}, x\right) \rightarrow 0$ (cf. [16, p. 200]). When $M$ is regarded as a $\mathrm{JBW}^{*}$-triple, we have $d_{\psi}(a, b)=\|a-b\|_{\psi}$.

The following lemma is a verbatim extension of [7, Lemmma 3] to the setting of JBW*algebras.

LEMMA 2.4. Let $M$ be a $J B W^{*}$-algebra with a faithful positive normal functional $\psi$. Let $\left(\phi_{n}\right)$ be a norm bounded sequence in $M_{*}$ and let $\Delta$ be the anti-compactness set of $\left(\phi_{n}\right)$ with respect to $\psi$, considering $M$ as a JBW*-triple. Then $\left(\phi_{n}\right)$ is relatively weakly compact in $M_{*}$ if and only if $\Delta=\emptyset$.

We sketch the main ideas of the proof for completeness. We have already shown that $\Delta=\emptyset$ implies $\left(\phi_{n}\right)$ being relatively weakly compact in $M_{*}$ (cf. Remark 2.3).

To prove the necessity we suppose, contrary to our claim, that $\Delta \neq \emptyset$. There is no loss of generality in assuming $\|\psi\|=1$. Let $c \in \Delta$. Then for each $k \in N$ there exists a tripotent $e_{k} \in M$ satisfying $\left\|e_{k}\right\|_{\psi}<2^{-k}$ and the set

$$
\left\{m \in N \text {; there exists } u \in \operatorname{Tri}(M) \text { with } u \leq e_{k} \text { and }\left|\phi_{m}(u)\right| \geq c\right\}
$$

is infinite. Thus $\left(e_{k}\right)$ is a bounded sequence in $M$ satisfying

$$
d_{\psi}\left(e_{k}, 0\right)=\left\|e_{k}\right\|_{\psi} \rightarrow 0 .
$$


Since $\psi$ is a faithful normal state of $M$, and the strong*-topology of $M$ is determined by the metric $d_{\psi}$, we deduce that $\left(e_{k}\right)$ tends to zero in the strong*-topology of $M$.

Since, by assumptions, $\left(\phi_{n}\right)$ is relatively weakly compact, by Theorem 1.1 there exist norm-one functionals $\varphi_{1}, \varphi_{2} \in M_{*}$ and $\delta>0$ satisfying that for every $x \in M$ with $\|x\| \leq 1$ and $\|x\|_{\varphi_{1}, \varphi_{2}}<\delta$ we have $\left|\phi_{n}(x)\right| \leq c / 2$ for all $n \in N$. Since $\left(e_{k}\right) \rightarrow 0$ in the strong*topology, there exists $k_{0} \in N$ such that for each $k \geq k_{0}$ we have $\left\|e_{k}\right\|_{\varphi_{1}, \varphi_{2}}<\delta$. Let $k \geq k_{0}$. It is not hard to see that (from the orthogonality of $u$ and $e_{k}-u$ ) for each tripotent $u \leq e_{k}$ we have $\|u\|_{\varphi_{1}, \varphi_{2}} \leq\left\|e_{k}\right\|_{\varphi_{1}, \varphi_{2}}<\delta$. Consequently, $\left|\phi_{n}(u)\right| \leq c / 2$ for all $n \in N$, which contradicts (3).

Having the above facts in mind, the proof of [7, Proposition 4] can be slightly adapted to prove the following result.

Proposition 2.5. Let $M$ be a $J B W^{*}$-algebra with a faithful positive functional $\psi$. Let $\left(\phi_{n}\right)$ be a norm bounded sequence in $M_{*}$. Then, for every $\varepsilon>0$, there exists a projection $p \in M$ such that $\psi(p)<\varepsilon$ and a subsequence of $\phi_{n},\left(\beta_{n}\right)$, such that the sequence $\left(\beta_{n}\right)$ restricted to $P_{2}(1-p)(M)$ is relatively weakly compact.

Let $\varphi$ be a norm-one functional in the predual of a $\mathrm{JBW}^{*}$-triple $W$. By [13, Proposition 2], there exists a unique tripotent $e=e(\varphi) \in W$ such that $\varphi=\varphi P_{2}(e)$ and $\left.\varphi\right|_{W_{2}(e)}$ is a faithful normal state of the $\mathrm{JBW}^{*}$-algebra $W_{2}(e)$. This unique tripotent $e=e(\varphi)$ is called the support tripotent of $\varphi$.

We can now state an analogue of [7, Theorem 8] in the setting of $\mathrm{JBW}^{*}$-triples.

THEOREM 2.6. Let $W$ be a JBW*-triple. Let $\varphi$ be a norm-one element in $W_{*}$ and $\left(\phi_{n}\right)$ a norm bounded sequence in $W_{*}$. Then, for each $1>\varepsilon>0$, there exists a tripotent $e \in W$ such that $\|e\|_{\varphi}>1-\varepsilon$ and a subsequence $\left(\phi_{\sigma(n)}\right)$ such that $\left(\left.\phi_{\sigma(n)}\right|_{W_{2}(e)}\right)$ is relatively $\sigma\left(\left(W_{2}(e)\right)_{*}, W_{2}(e)\right)$-compact in $\left(W_{2}(e)\right)_{*}$.

Proof. Let $s=s(\varphi)$ be the support tripotent of $\varphi$. Let $\varepsilon>0$. By Proposition 2.5, there exists a projection $p \in W_{2}(s)$ such that $\varphi(p)<\varepsilon$ and a subsequence $\left(\phi_{\sigma(n)}\right)$ such that $\left(\phi_{\sigma(n)}\right)$ restricted to $P_{2}(s-p)(W)$ is relatively weakly compact. We take $e=s-p$ to obtain the desired statement.

Replacing [7, Theorem 8] by Theorem 2.6 in the proof of [7, Corollaries 9, 10] we obtain the following

COROLLARY 2.7. Let $\varphi$ be a norm-one functional in the predual of a JBW*-triple $W$. Let $\left(\phi_{n}\right)$ be a norm bounded sequence in $W_{*}$ and let $s=s(\varphi)$ be the support tripotent of $\varphi$. Then there exists a sequence of tripotents $\left(e_{k}\right)$ (with $e_{k} \leq s$ for each $k \in N$ ) which converges in the strong*-topology to $s$ and a subsequence of $\left(\phi_{n}\right),\left(\phi_{\sigma(n)}\right)$, such that

$$
\lim _{n \rightarrow+\infty} \phi_{\sigma(n)} P_{2}\left(e_{k}\right)(x)
$$

exists for each $x \in W$. 


\section{REFERENCES}

[ 1 ] M. D. Acosta And A. M. Peralta, An alternative Dunford-Pettis property for JB*-triples, Q. J. Math. 52 (2001), 391-401.

[2 2 C. A. Akemann, The dual space of an operator algebra, Trans. Amer. Math. Soc. 126 (1967), 286-302.

[ 3 ] C. A. Akemann, P. G. Dodds And J. L. B. Gamlen, Weak compactness in the dual space of $C^{*}$-algebra, J. Functional Analysis 10 (1972), 446-450.

[4] T. BARTON AND Y. Friedman, Grothendieck's inequality for JB*-triples and applications, J. London Math. Soc. (2) 36 (1987), 513-523.

[ 5 ] T. BARton And Y. Friedman, Bounded derivations of JB*-triples, Quart. J. Math. Oxford Ser. (2) 41 (1990), 255-268.

[6] T. Barton And R. M. Timoney, Weak*-continuity of Jordan triple products and its applications, Math. Scand. 59 (1986), 177-191.

[ 7 ] J. K. BRooks, K. Saitô and J. D. M. Wright, A bounded sequence of normal functionals has a subsequence which is nearly weakly convergent, J. Math. Anal. Appl. 276 (2002), 160-167.

[ 8 ] L. J. BunCE, Norm preserving extensions in JBW*-triple preduals, Q. J. Math. 52 (2001), 133-136.

[ 9 ] C.-H. ChU AND B. Iochum, Weakly compact operators on Jordan triples, Math. Ann. 281 (1988), 451-458.

[10] C.-H. ChU And P. Mellon, The Dunford-Pettis property in JB*-triples, J. London Math. Soc. (2) 55 (1997), 515-526.

[11] S. DineEN, The second dual of a JB*-triple system, Complex analysis, functional analysis and approximation theory (Campinas, 1984), 67-69, North-Holland Math. Stud. 125, North-Holland, Amsterdam, 1986.

[12] C. M. EdWARDS, On Jordan W*-algebras, Bull. Sci. Math. (2) 104 (1980), 393-403.

[13] Y. FRIEdman AND B. Russo, Structure of the predual of a JBW*-triple, J. Reine Angew. Math. 356 (1985), $67-89$.

[14] H. Hanche-Olsen And E. Størmer, Jordan operator algebras, Monogr. Stud. Math. 21, Pitman, Boston Mass. 1984.

[15] G. HoRN, Characterization of the predual and ideal structure of a JBW*-triple, Math. Scand. 61 (1987), $117-$ 133.

[16] B. Iochum, Cônes autopolaires et algebres de Jordan, Lecture Notes in Math. 1049, Springer-Verlag, Berlin, 1984.

[17] H. JARCHOW, Weakly compact operators on $C(K)$ and $C^{*}$-algebras, Functional analysis and its applications (Nice, 1986), 263-299, ICPAM Lecture Notes, World Sci. Publishing, Singapore, 1988.

[18] H. JARCHOW, On weakly compact operators on $C^{*}$-algebras, Math. Ann. 273 (1986), 341-343.

[19] W. KAUP, A Riemann mapping theorem for bounded symmetric domains in complex Banach spaces, Math. Z. 183 (1983), 503-529.

[20] O. Loos, Bounded symmetric domains and Jordan pairs, Math. Lectures, University of California, Irvine (1977).

[21] A. M. Peralta and A. Rodríguez Palacios, Grothendieck's inequalities for real and complex JBW*triples, Proc. London Math. Soc. (3) 83 (2001), 605-625.

[22] K. SAITô, On the preduals of $W^{*}$-algebras, Tôhoku Math. J. (2) 19 (1967), 324-331.

[23] S. SAKAI, C*-algebras and W*-algebras, Ergeb. Math. Grenzgeb. 60, Springer-Verlag, New York-Heidelberg, 1971.

[24] M. TAKESAKI, On the conjugate space of operator algebra, Tôhoku Math. J. (2) 10 (1958), 194-203.

[25] M. TAKESAKI, Theory of operator algebras I, Springer-Verlag, New York-Heidelberg, 1979.

[26] J. D. M. WRight, Jordan C*-algebras, Michigan Math. J. 24 (1977), 291-302. 
DePartamento de AnÁlisis MATEMÁtico

FACUlTAD DE CiEnCIAS

UNIVERSIDAD DE GRANADA

18071 GRANADA

SPAIN

E-mail address: aperalta@ugr.es 